\title{
Anomalous Diffraction of Gram-positive Bacteria
}

\author{
By P. J. ORIEL \\ Biochemical Research Laboratory, The Dow Chemical Company, \\ Midland, Michigan 48640, U.S.A.
}

(Accepted for publication 4 April I967)

SUMMAR Y

\begin{abstract}
Application of the anomalous diffraction theory of van de Hulst to turbidity measurements of Gram-positive bacteria is discussed. It is shown that this method is capable of detecting characteristic morphological changes during growth. Comparison with the cell mass method and direct observations of Micrococcus freudenreichii suggest that these changes are primarily related to cluster size. Initial application of the method in the study of osmotic change in Bacillus megaterium is described.
\end{abstract}

\section{INTRODUCTION}

Although it has been shown that at least some bacteria fit the homogeneous spherical models for which simple scattering theories are available (Koch, 196I; Petukhov, 1965), use of light scattering in studying bacterial growth and transport has been largely limited to empirical correlation of scattering properties with other physical measurements. The difficulties in applying scattering theory lie in the need for extrapolation of scattering data to zero concentration, determination of concentration and refractive index of particles, and the necessity to use cumbersome numerical theoretical solutions.

In this paper we wish to describe the application of the anomalous diffraction theory of van de Hulst (1946, 1947, 1962) in studying some Gram-positive bacteria. It is shown that by use of this simple approximation coupled with curve fitting by computer, kinetic studies of bacterial morphology can readily be accomplished, eliminating determination and extrapolation of concentration.

\section{THEORY}

For spherical particles with dimensions comparable to the wavelength of the measuring radiation, complete Mie theory (Mie, 1908) is generally required to account for multipole scattering and intraparticle interference. Van de Hulst (1946, 1947, 1962) has shown, however, that for particles with refractive index close to that of the medium, assumption of negligible ray deviation and back scattering can be made. The light perturbation can then be described by diffraction and phase lag in the spherical particle. The resultant efficiency factor is described by the closed expression

where

$$
Q_{\text {oxt. }}=2-4 / \rho \sin \rho+4 / \rho^{2}(\mathrm{I}-\cos \rho)
$$

$$
\rho=\frac{4 \pi\left(n_{p}-n_{0}\right) a}{\lambda_{0}}
$$


$n_{p}$ is the refractive index of the particle, $n_{0}$ is the refractive index of the solvent, $a$ is the particle radius, $\lambda_{0}$ is the wavelength in vacuo. At low $\rho$, van de Hulst (1962) has pointed out that equation (I) is asymptotic with the Jobst equation (Jobst, I925).

$$
Q_{\text {ext. }}=\frac{1}{2} \rho^{2} \text {. }
$$

The extent of the approximation introduced by use of equation (I) rather than Mie theory is seen in Fig. I. Mie data (Pangonis, Heller \& Jacobsen, 1957) for

$$
\frac{n_{p}}{n_{0}}=1 \cdot 05
$$

is shown, as this refractive index most closely approximates that found in bacteria (see below).

The extinction efficiency factor is related to the apparent extinction coefficient $\gamma$ by

$$
\gamma=N \pi a^{2} Q_{\text {ext. }}
$$

where $N$ is the number of particles per unit volume of solution. Lewis \& Lothian (1954) showed that if particles are large enough to deviate from the Jobst equation, plots of experimental turbidity with $\mathrm{I} \lambda$ and $\gamma$ with $\rho$ (equation (4)) could be compared. By expanding the ordinate $\rho$ of the theoretical curve so that maxima and minima were overlapped with the experimental curve, $\rho$ values corresponding to given wavelengths were obtained. From these, values of $4 \pi\left(n_{p}-n_{0}\right) a$ (we shall call this product $B$ ) were calculated using equation (2). Van de Hulst (1947) has shown that the best value of radius $\left(a_{\mathrm{obs} .}\right)$ to adopt for a heterodisperse system such as this is given by the expression

$$
a_{\text {obe. }}=-o \frac{\int_{0}^{\rho_{0}} f(a) a^{3} d a}{\int_{0}^{\infty} f(a) a^{2} d a},
$$

where $f(a)$ is the number of particles with size between $a$ and $a+d a$. The studies of Lewis \& Lothian were made on barium sulphate suspensions and Lycoperdon pyriforme spores. These particles are large enough for the first maximum in Fig. I to be obtained with visible radiation. With the smaller size of most bacteria, this maximum is not reached with visible and near ultraviolet wavelengths, requiring non-linear curve fitting. In this work the difficulty in fitting is circumvented by the use of electronic computation. Because of the difficulty in separating refractive index and size effects in growing bacterial systems, $\boldsymbol{B}$ values will be reported as such, and corollary experiments to separate the contributions discussed.

It should be noted that the turbidity dispersion method has also been applied using numerical Mie data (see, for example, Barns \& LaMer, 1946; Heller, Bhatnagar \& Nakagaki, 1962; Fikhman, 1963) but refractive index must be assumed, and wavelengths of measurement are dictated by the available numerical data, making the method somewhat inflexible for biological particles.

\section{Monodisperse latexes}

EXPERIMENTAL.

Monodisperse polystyrene latexes were obtained from the Bioproducts Department of The Dow Chemical Company. Sizes of the samples had been determined using 
electron microscopy. The latexes were diluted with glass-distilled water and measured without further treatment.

\section{Culture media and organisms}

The principal requirement of culture media used in growth experiments is that the media be relatively transparent in the wavelength range of measurement. Chemically defined media without cofactors were used. To obtain vigorous growth it was found necessary to add small amounts of Difco-brain-heart infusion broth (BHI). The basal medium consisted of $0.3 \% \mathrm{NH}_{4} \mathrm{H}_{2} \mathrm{PO}_{4}, 0.7 \% \mathrm{~K}_{2} \mathrm{HPO}_{4}$, and $5 \times 10^{-4} \% \mathrm{FeSO}_{4}$; the $\mathrm{pH}$ was $7 \cdot \mathrm{I}$. Organisms and media used were:

Micrococcus freudenreichii (ATCC 407). Basal medium with 0.25\% Difco-Casamino acids and $0 . \mathrm{II} \% \mathrm{BHI}$.

Micrococcus candidus (ATCC 8425). Basal medium with $0.5 \%$ glucose and $0.2 \%$ BHI.

Bacillus megaterium (ATCC 13632). $3 \cdot 7 \%$ BHI.

\section{Growth conditions}

Two hundred and fifty $\mathrm{ml}$. volumes in $500 \mathrm{ml}$. Erlenmeyer flasks were used. Flasks were vigorously shaken in a Gyrotropic shaker (New Brunswick Scientific) at $30^{\circ}$. Micrococcus freudenreichii and $M$. candidus were inoculated from $24 \mathrm{hr}$ cultures at concentrations of $1 \times 10^{7}$ and $2.5 \times 10^{6}$ organisms $/ \mathrm{ml}$. respectively. Bacillus megaterium was grown overnight, harvested and washed twice with distilled water, and gently resuspended in distilled water.

\section{Spectrophotometer}

Turbidity measurements were obtained using a Beckman DK-2A spectrophotometer. This instrument is well suited to turbidity measurements, as the distance between cell and photomultiplier is long, limiting measurements to a small solid angle around zero. Turbidity was found to follow the Beer-Lambert Law within experimental error at all wavelengths below an apparent optical density of $\mathrm{I} \cdot 0$. Errors due to slit width variation during scanning were estimated to be $1 \%$ or less. This was determined by noting the total change in slit width throughout the scan, and manually varying the slit by this amount at the extreme wavelengths by changing the sensitivity or amplifier gain. For more complete discussion of the instrumental requirements for turbidity measurements see Heller \& Tabibian (1957).

\section{Spectrophotometer measurements}

The reference cell contained fresh medium. Periodic checks were made to determine that the presence of bacteria was not altering the absorption of the medium. This was done by centrifuging the culture at intervals during the growth cycle and scanning the supernatant against fresh medium. It was found in this way that Micrococcus candidus excretes an ultraviolet-absorbing material during growth, and measurements on this organism were confined to the 600 to $400 \mathrm{~m} \mu$ region.

Rather than changing the environment of the organisms during growth by dilution, turbidity was reduced below I by using cells of $10,5,2$, and I mm. path lengths. Checks were routinely made of $B$ values upon changing path lengths during the runs. 
Samples were scanned immediately after sampling to avoid temperature-induced changes in refractive index.

\section{Refractive increment measurements}

Suspensions for refractive increment measurements were made by centrifuging overnight cultures, washing twice with distilled water, followed by centrifugation of the suspension at $17,000 \mathrm{rev}$. $/ \mathrm{min}$. in a Sorvall SS-34 head for $30 \mathrm{~min}$. The supernatant was poured off and the tubes with wet organisms recentrifuged in the same manner. This centrifugation removed much of the occluded water contained in the wet mass. The organisms were then weighed and resuspended for measurement. Refractive index measurements were made in a Phoenix BS 300 differential refractometer at $546 \mathrm{~m} \mu$. Usually five dilutions of a $1 \%$ (wet w/v) stock were measured. Although refractive index is quite temperature-dependent, differential refractive indices are much less dependent. Hence, solutions were equilibrated and run at ambient temperature (approximately $23^{\circ}$ ).

\section{Microscopy}

Direct measurements of the bacterial cluster diameters were performed using an American Optical Phase Star Microscope with Polaroid camera attachment. Samples of the growing culture were dried and stained with crystal violet after which pictures were taken at a magnification of 970 . These were subsequently enlarged for diameter measurements. Both minimum and maximum dimensions were recorded for Micrococcus freudenreichii, as most clusters were not perfectly spherical.

\section{Computations}

Electronic computations utilized a Burroughs B 5500 computer. Input consisted of turbidity measurements at $25 \mathrm{~m} \mu$ intervals from either 500 to $350 \mathrm{~m} \mu$ or from 600 to $400 \mathrm{~m} \mu$. A range of $B$ values with specified increments in $B$ was set for trial and error fitting. For a given spectrum the selection of best fit (and $B$ value) was based on a minimum in

$$
S S \text { (sum of squares) }=\Sigma_{i}\left[T\left(\lambda_{i}\right)-F \gamma\left(\lambda_{i}\right)\right]^{2},
$$

where $T\left(\lambda_{i}\right)$ is the turbidity measured at wavelength $i, \gamma\left(\lambda_{i}\right)$ is the extinction calculated at wavelength $i$ for a specified $B$ according to equation (4), and $F$ is the normalization factor

$$
F=\frac{T\left(\lambda_{l}\right)}{\gamma\left(\lambda_{l}\right)}
$$

equating theoretical and experimental turbidity at one wavelength (usually the lowest wavelength measured). This factor incorporates the $4 \pi N a^{2}$ in equation (4), eliminating the need for concentration determination. Figure 2 shows the fit of points for the best $B$ value with an experimental turbidity dispersion (Micrococcus freudenreichii). It should be noted that in theory turbidity data from only two wavelengths are required for the method, but inclusion of more data allows a better determination of the degree of fit obtained. 


\section{Monodisperse latexes}

RESULTS

As a preliminary trial of the method, turbidity dispersions were taken of latexes with sizes of 1.305 and $0.796 \mu$. Although these particles have a uniform spherical size, the refractive index of latex relative to water $\left(n_{p} / n_{0}=\mathrm{I} \cdot 20\right)$ is somewhat greater than that desirable for use of the anomalous diffraction theory. The particle diameters for the two latexes were estimated from the turbidity measurements to be $\mathrm{I} \cdot 3 \mathrm{IO}$ and $0.715 \mu$.

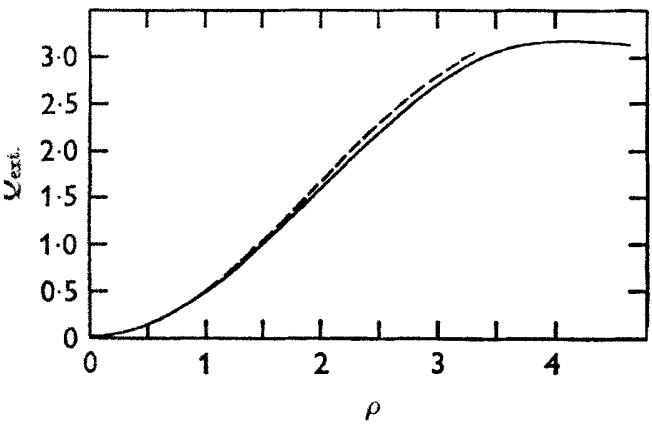

Fig. I

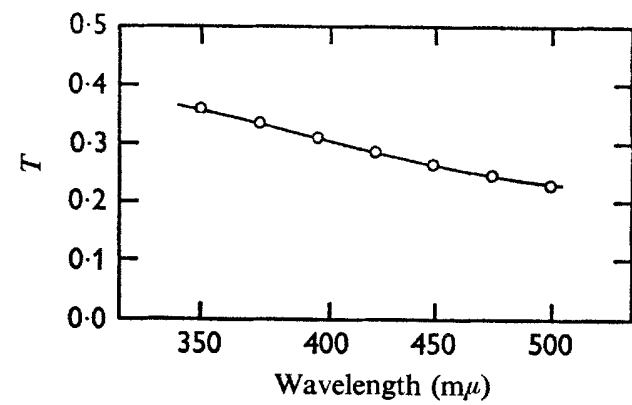

Fig. 2

Fig. I. Comparison between the extinction efficiency factor for anomalous diffraction theory (-) and Mie theory (--) for $n_{p} / n_{o}=1 \cdot 05$. (Mie data from Pangonis, Heller \& Jacobsen, 1957.)

Fig. 2. Comparison of best fit with experimental turbidity dispersion of Micrococcus freudenreichii. (-), experimental; (O), best theoretical fit.

\section{Batecrial growth}

The principal question to be answered in investigating the use of this technique is to what extent is the method sensitive to bacterial morphological change during growth. Since both Micrococcus freudenreichii and $M$. candidus grow in cell clusters, it was of interest to determine the uniformity of cluster size in different cultures, and ask whether cluster breakup was coincident with cell division. To answer these questions, samples of the growing cultures were scanned at 20-30 min. intervals from inoculation into the start of the stationary phase. Results for two cultures each of Micrococcus candidus and $M$. freudenreichii are shown in Figs 3 and 4 . The general growth was measured by turbidity at $550 \mathrm{~m} \mu$ as shown in Fig. 5. It is seen that the profile of $B$ with time is markedly different for the two bacteria. For $M$. candidus a rather rapid increase in $B$ is seen after inoculation with subsequent drop near the centre of the log phase. In profile this curve resembles the classic cell size curve for single bacteria (Thimann, 1964). With $M$. freudenreichii, the increase is more gradual, continuing until the end of the log phase with a drop thereafter. In a series of six runs with different cultures under the same growth conditions, although the initial $B$ values for $M$. freudenreichii varied between 910 and $1090 \mathrm{~m} \mu$, the maximum in $B$ was found to be a more uniform value of $1280 \pm 20 \mathrm{~m} \mu$.

\section{Comparison with the cell mass method and direct observation}

Because of the difficulty in making direct measurements of refractive index with cell growth (see below), independent methods of measuring cluster size were carried 


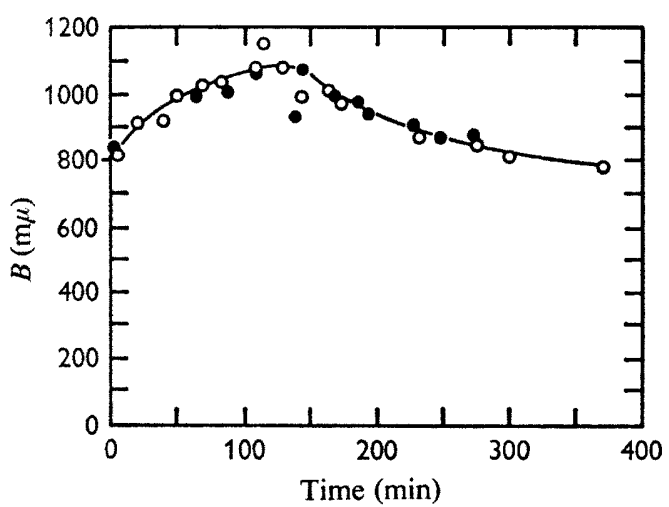

Fig. 3

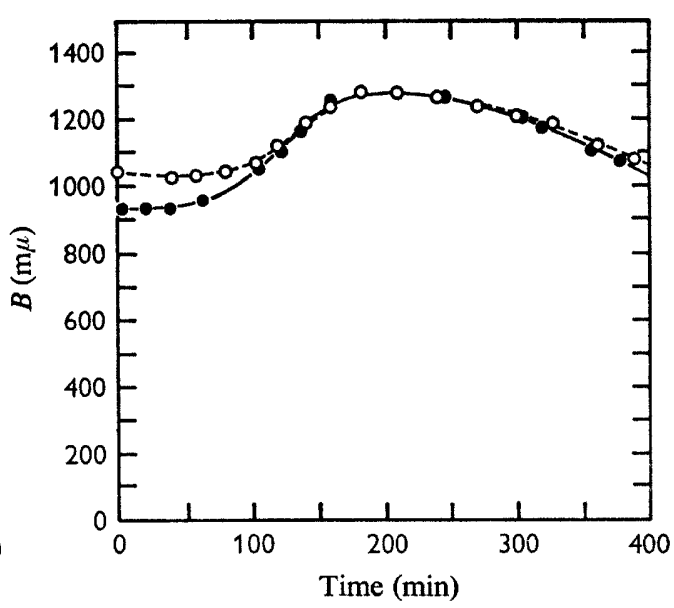

Fig. 4

Fig. 3. Profile of $B$ change during growth cycle of Micrococcus candidus.

$(O-O)$, culture $\mathrm{I}$; $(\mathrm{O}-\mathrm{O})$, culture 2 .

Fig. 4. Profile of $\boldsymbol{B}$ change during growth cycle of Micrococcus freudenreichii.

(O-O), culture $\mathrm{I}$; $(\mathrm{O}-\mathrm{O})$, culture 2 .

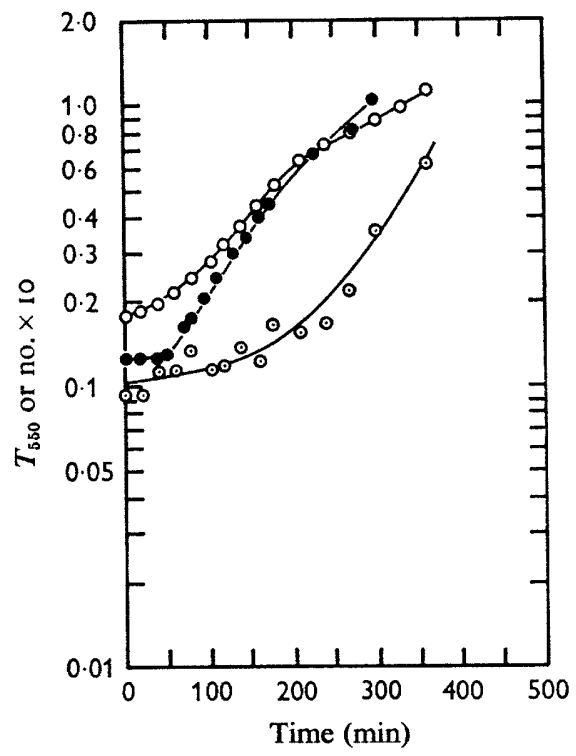

Fig. 5. $T_{550}$ and viable count measurements during growth cycles. $(\mathrm{O}-\mathrm{O}), T_{550}$ of $M$. freudenreichii; ( $\odot), T_{550}$ of $M$. candidus; $(\odot-\odot)$, viable count of $M$. freudenreichii.

out. The standard microbiological method for measuring relative cell mass or size consists of dividing turbidity at a single wavelength by the number of viable organisms. Koch (196I) has pointed out that assuming that $n_{p}-n_{0}$ remains constant and if the bacteria follow the Jobst equation, such a measurement will actually be proportional to $a^{4}$ (for anomalous diffraction, $B^{4}$ ). If we assume that at high wavelengths (low $\rho$ ) the turbidity is not far removed from the Jobst equation, a direct comparison between 
the anomalous diffraction and cell mass method can then be made. This is shown in Fig. 6 for Micrococcus freudenreichii, using $B^{4}$ values (from Fig. 4) and $T_{550}$ and viable count data (Fig. 5). Since the cell mass method is only relative, both sets of data have been normalized with regard to the initial reading. It is seen that although the anomalous diffraction method yielded much better precision, good general agreement is obtained.

Direct measurements of cluster size were carried out for Micrococcus freudenreichii during a growth experiment similar to that described above. $B$ values were observed and slides taken of the growing culture as described in the experimental section. Pictures from 3 and $62 \mathrm{~min}$. were used to characterize the low $B$ region in the lag phase and pictures taken at 180 and $210 \mathrm{~min}$. were used to characterize the bacteria at the maximum $B$ value. Measurements from anomalous diffraction yielded an observed diameter of $3.2 \mu$ in the lag phase and $4.3 \mu$ near $200 \mathrm{~min}$., a $34 \%$ increase. Direct measurement yielded an average diameter in the lag phase of $2.3 \mu$, and an average diameter near $200 \mathrm{~min}$. of $2.9 \mu$, a $26 \%$ increase. The differences between the 75 measurements from the lag region and the 44 measurements from the $200 \mathrm{~min}$. region were statistically analysed using the ' $t$ ' test, which showed a confidence value of $>99 \%$ for the observed difference.

Micrococcus candidus was observed to grow in chain-like clusters from I to 30 or more cells. Measurement of a slide taken 3 min. after inoculation revealed a cell width of approximately $0.5 \mu$ and an average end-to-end chain length of approximately $5 \mu$. The value of $2 a_{\text {obs. }}$ at this time was $3 \cdot 2 \mu$.

\section{Refractive index}

Measured refractive indices of the cells in the stationary phase are shown in Table I. Because of possible persistent error due to adsorbed or occluded water these values

Table I. Refractive index difference values for some Gram-positive bacteria

\begin{tabular}{lc}
\multicolumn{1}{c}{ Organism } & $n_{p}-n_{0}$ \\
Micrococcus freudenreichii & 0.047 \\
M. candidus & 0.040 \\
Bacillus megaterium & 0.048
\end{tabular}

must be regarded as approximate. Some comparison can be made with the results of Barer \& Joseph (1958) and Fikhman (1964) using phase-immersion refractometry. Although the organisms used in this work were not measured, these investigators obtained $\left(n_{p}-n_{0}\right)$ values for various bacteria from 0.045 to 0.080 , and 0.037 to 0.067 , respectively.

\section{TRANSPORT EXPERIMENTS}

In an attempt to determine the value of anomalous diffraction in transport experiments, experiments similar to those described by Avi-Dor, Kuczynski, Shatzberg \& Magar (1956) were carried out with Bacillus megaterium. In this case washed bacteria were transferred from distilled water to $\mathrm{M}-\mathrm{NaCl}$ and both turbidity, at $550 \mathrm{~m} \mu$, and anomalous diffraction observed with time. The results are shown in Fig. 7. Although the anomalous diffraction method allows the advantage that absolute $B$ values can be determined from such kinetics, again for comparison with the single wavelength 
measurements, the data is normalized with respect to the bacteria in water. It is seen that the kinetics differ, but that both methods reflect the initial increase and slow decrease reported by Avi-Dor et al. (1956).

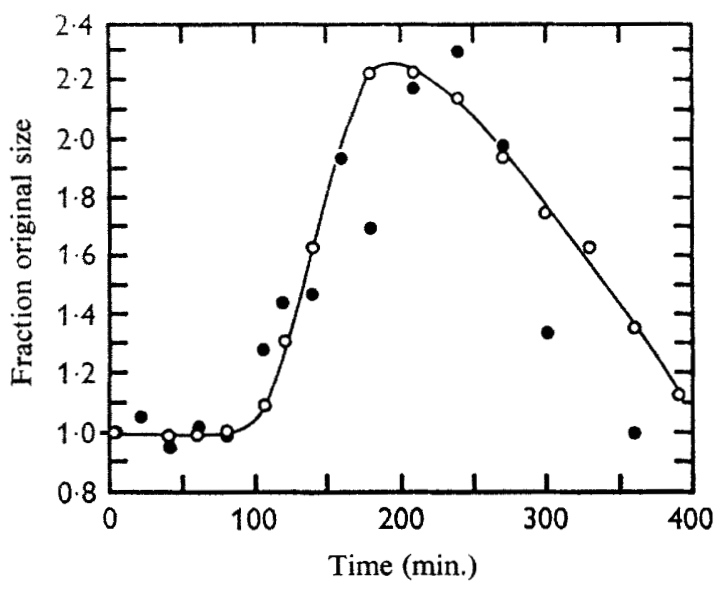

Fig. 6

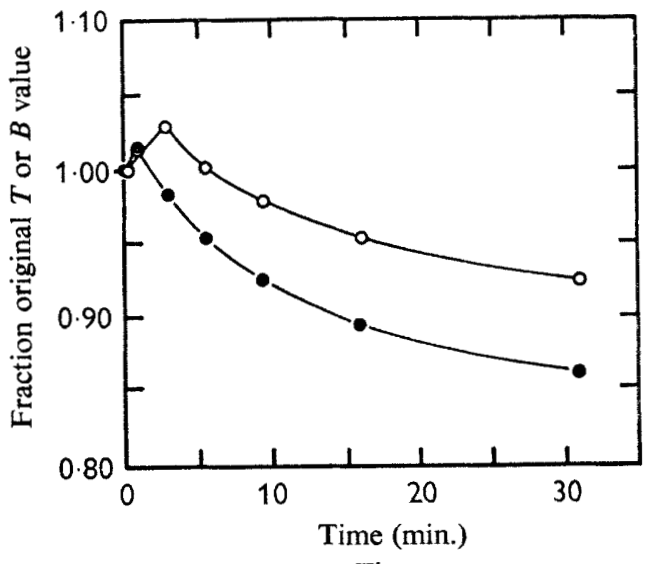

Fig. 7

Fig. 6. Comparison of cell mass and anomalous diffraction methods for Micrococcus freudenreichii. $(\mathrm{O}-\mathrm{O}), B^{4} ;\left(O_{-O}\right), T_{550} /$ no. Both curves are normalized with respect to the first point.

Fig. 7. Transport kinetics of Bacillus megaterium following immersion in $\mathrm{M}-\mathrm{NaCl} .(\mathrm{O}-\mathrm{O}$ ), $B$ values from anomalous diffraction; $(-0), T_{550}$ values. Both curves normalized with respect to zero time.

\section{DISCUSSION}

Neither the latexes nor the bacteria tested completely meet the theoretical requirements of the anomalous diffraction theory. The requirement for low particle refractive index relative to that of the solvent is only approximated with the former, and the requirement for spherical shape is only approximated with the latter. None the less, good agreement is attained with the latexes, especially with the $\mathrm{I} \cdot 305 \mu$ particle diameter. This size falls in the $\rho$ region where deviation between the Mie and anomalous diffraction theories for $n_{p} / n_{0}=\mathrm{I} \cdot 20$ is not as large as with smaller sizes.

Because the roughly spherical clusters of Micrococcus freudenreichii most closely meet the shape requirement, this bacterium was chosen for principal emphasis. The changes in cell mass and directly observed diameter with growth are in good agreement with the changes in $B^{4}$ and $2 a_{\text {obs. }}$, respectively. This indicates that the profile of $B$ with growth cycle primarily reflects size changes. This is reasonable, since Barer \& Joseph (I958) have pointed out that the macromolecules in bacteria have similar refractive indices. As a result, large changes in $n_{p}-n_{0}$ would reflect large changes in water content of the cytoplasm which would not be expected during growth. The data also indicate that cell division and cluster break-up are independent phenomena for this bacterium. It is interesting to speculate that the more uniform $a_{\text {obs. }}$ of different cultures at the end of the log phase may be related to a control mechanism for cluster breakup.

The agreement in absolute size is not as good with Micrococcus freudenreichii as with the latexes, probably due to either errors in refractive increment determination due to adsorbed water or the high weighting of the size distribution noted above. 
Since the experimental fits are good throughout the wavelengch range, we conclude that the refractive index dispersion and bacterial light absorption do not contribute significant errors to the size determination.

In spite of the complicated geometry of Micrococcus candidus clusters, good fits of turbidity dispersions were obtained using anomalous diffraction theory. The $2 a_{\text {obs. }}$. value lies between the cluster diameter and end-to-end distance. It will be interesting to see if this holds true for other rod-shaped clusters, for which simple light-scattering theories are not available.

Studies of bacteria exposed to a new osmotic environment have proved important in studies of active transport (see, for example, Packer \& Perry, 196I; Rogers \& Yu, 1963). The difficulty in interpreting such measurements is that in this case, refractive index changes from solvent transport are expected to be important, making it difficult to separate the effect of solute and solvent. We feel that since anomalous diffraction weights refractive index and size differently than single wavelength turbidity, use of both methods simultaneously may be useful in separating solute and solvent transport. Due to complications in interpreting $a_{\text {obs. }}$ with rods (van de Hulst, 1962), an attempt to perform separations of this sort will better await more detailed experiments utilizing spherical bacteria.

In conclusion, although more work needs to be done to clearly define the potential of the method, the experiments above show that the anomalous diffraction technique can readily be used for studies of bacterial morphology. It is possible that the method can also be utilized in studies of mitochondria and red blood cells as well.

The expert technical assistance of Miss Janice Wilder is gratefully acknowledged.

\section{REFERENCES}

Avi-Dor, Y., Kuczynski, M., Shatzberg, G. \& Magar, J. (I956). Turbidity changes in bacterial suspensions: kinetics and relation to metabolic state. J. gen. Microbiol. 14, 76.

BARER, R. \& JOSEPH, S. (1958). Concentration and mass measurements in microbiology. J. appl. Bact. 2I, 146.

Barnes, M. D. \& LAMER, V. K. (1946). Monodispersed hydrophobic colloidal dispersions and light scattering properties. II. Total scattering from transmittance as a basis for calculation of particle size and concentration. J. Colloid Sci. r, 79.

FIKHMAN, B. A. (1963). Light scattering by bacteria in the visible region of the spectrum. Biofizika 8 , 380 .

Fikhman, B. A. (1964). Refractometry of Bacteria. Ph.D. Thesis cited by Petukhov (1965).

Heller, W. \& TABIBIAN, J. (1957). Experimental investigations on the light scattering of colloidal spheres. II. Sources of error in turbidity measurements. J. Colloid Sci. 12, 25.

Heller, W., Bhatnagar, H. L. \& Nakagaki, M. (1962). Theoretical investigations of the light scattering of spheres. XIII. The 'wavelength exponent' of differential turbidity spectra. J. chem. Phys. 36, 1163.

VAN DE Hulst, H. C. (1946). Optics of spherical particles. Res. Astron. Obs. D'Utrecht rr, pt. r.

vAN DE HULST, H. C. (1947). The solid particles in interstellar space. Res. Astron. Obs. D'Utrecht II, pt. 2.

vAN DE Hulst, H. C. (1962). Light Scattering by Small Particles. New York: John Wiley and Sons.

JoBst, G. (1925). Diffuse Strahlung dielektrischen Kugeln im Grenzfalle, wenn das Kugelmaterial und umgebendes Medium fast gleiche Brechungsindices haben. Annln Phys. 78, 157.

KoCH, A. (I96I). Some calculations on the turbidity of mitochondria and bacteria. Biochim. biophys. Acta 51, 429. 
Lewis, P. C. \& Lothian, G. F. (1954). Photoextinction measurements on spherical particles. Br. J. appl. Phys., Nottingham Conf. Suppl. C2.

MIE, G. (1908). Beiträge zur Optik trüber Medien, speziell kolloidaler Metallösungen. Annln Phys. 25, 337.

PACKer, L. \& Perry, M. (1961). Energy-linked light scattering changes in Escherichia coli. Archs Biochem. Biophys. 95, 379.

Pangonis, W. J., Heller, W. \& Jacobsen, A. W. (1957). Tables of Light Scattering Functions. Detroit: Wayne State University Press.

Petukhov, V. G. (1965). The possibility of applying the Mie theory to the scattering of light by suspensions of spherical bacteria. Biofizika ro, 993.

Rogers, D. \& YU, S. (1963). Turbidity changes during glucose permeation in Escherichia coli. J. Bact. $85,1141$.

Thimann, K. V. (1964). The Life of Bacteria. New York: MacMillan. 